\title{
The Determinants of Fall in the Elderly at the Geriatric Center of Ouakam (Senegal)
}

\author{
Ousseynou Ka ${ }^{1, *}$, Boris Arthur Aboua Ghébi ${ }^{2}$, Abdoul Aziz Ndiaye ${ }^{1}$, Atoumane Faye ${ }^{2}$, \\ Mamadou Makhtar Mbacké Léye ${ }^{2}$, Mamadou Diallo ${ }^{3}$, Souhaibou Ndongo ${ }^{2}$ \\ ${ }^{1}$ Research Training Unit: Health and Sustainable Development of Alioune Diop University of Bambey, Bambey, Senegal \\ ${ }^{2}$ Faculty of Médicine, Cheikh Anta Diop University of Dakar, Dakar, Senegal \\ ${ }^{3}$ Department of Letters and Linguistics of Gaston Berger University of Saint Louis, Saint Louis, Senegal
}

Email address:

Ousseynou.ka@uadb.edu.sn (O. Ka), bborisarthur@yahoo.fr (B. A. A. Ghébi), abdoulaziz.ndiaye@uadb.edu.sn (A. A. Ndiaye), atoufayemi@yahoo.fr (A. Faye), mamadou.leye@yahoo.fr (M. M. M. Leye), siyaale@yahoo.fr (M. Diallo),

sndongo_medinterne@yahoo.fr (S. Ndongo)

${ }^{*}$ Corresponding author

\section{To cite this article:}

Ousseynou Ka, Boris Arthur Aboua Ghébi, Abdoul Aziz Ndiaye, Atoumane Faye, Mamadou Makhtar Mbacké Léye, Mamadou Diallo, Souhaibou Ndongo. The Determinants of Fall in the Elderly at the Geriatric Center of Ouakam (Senegal). World Journal of Public Health. Vol. 4, No. 1, 2019, pp. 24-29. doi: 10.11648/j.wjph.20190401.14

Received: March 17, 2019; Accepted: April 25, 2019; Published: May 27, 2019

\begin{abstract}
In Sénégal, the reality of falls in the elderly, despite their being a serious problem, are not well-known to the général public because the epidemiological data do not take them into account. This study aims at analyzing falls in elderly people who come for consultation at the gerontology and geriatrics center of Ouakam, focusing in particular on their frequency and their determinants. This was a descriptive cross-sectional study that was conducted from May 9 to July 10, 2016 at the Gerontology and Geriatrics Center. The population under investigation consisted of 210 patients with a female prédominance (61\%), an average age of 69 years, $72 \%$ married, $22 \%$ practiced physical activity, $54 \%$ had a normal weight, $58 \%$ took more of a medicine. The most common pathologies were visual acuity $(70 \%)$, arterial hypertension $(52 \%)$, osteoarthritis $(25 \%)$... The most prescribed drug classes were hypotensives $(33 \%)$, analgesics $(13 \%)$, antidiabetics drugs $(10 \%)$. The frequency of the falls in our population was $27 \%$ and they usually occurred at home $(65 \%)$. The determinants of the falls were soil barriers $(35 \%)$, loss of balance $(22 \%)$, wet surfaces $(16 \%)$, stairs $(8 \%) \ldots$ The number of medications taken per patient was only one factor statistically correlated with the number of falls. Bruising accounted for most of the mechanical complications (62\%) but all patients had developed a phobia for falling again. Recommendations have been made for better management of these falls.
\end{abstract}

Keywords: Falls, Elderly, Determinants, Geriatric Center, Senegal

\section{Introduction}

Falling is an event where a person inadvertently finds themselves on the ground or any other surface at a lower level than where they were previously. Falls in the elderly are frequent events. And yet, their epidemiological reality remains unknown to this day. Their prevalence and incidence are probably undervalued because they are often only counted when they justified medicalization. Thus, many falls without consequences are not taken into account in the statistical data; they are also quickly forgotten by the victims themselves during post-investigations. Falls are a global public health problem as they are the second leading cause of deaths by accident or death by unintentional injury $[1,2]$. Older people remain the most affected by this phenomenon. Thus any fall in an elderly person, even apparently innocuous, should be explored and managed as a potentially serious condition with possible risk of recurrence and complications [3]. The short-term severity of the fall may be due directly to the fall itself and in this case depends on the immediate consequences that can range from contusion to more severe trauma (dislocations, vertebral compression, cerebral hematomas or fractures) [4]. Mortality is even higher if there are co-morbidities or the patient has 
postoperative complications [5]. A fall can cause a real psychological trauma for the elderly. This results in reactions that may take the form of a loss of self-confidence, a deterioration of self-image or a feeling of insecurity [6]. The mechanism related to falling is most often multifactorial, involving intrinsic factors related to senescence and various defects, and to extrinsic factors as well [7, 8]. In Senegal, as elsewhere, the reality of falls among the elderly is not well known and the national epidemiological data do not take them into account despite their seriousness (disability, fractures especially of femoral neck...). The general objective of our study was to study falls, in particular their frequency and determinants in the elderly people who came for consultation at the gerontology and geriatrics center of Ouakam.

\section{Methods}

\subsection{Study Framework}

The survey was carried out in the district municipality of Ouakam (Dakar). Its population, which is estimated at 60000 inhabitants, is essentially young with a proportion of people aged 60 and over representing 6.5\% [9]. Most older people have no pension or health coverage. Their medico-social care is usually provided by the family. Health facilities in Ouakam include a municipal health center, a gerontology and geriatrics center, a military hospital and a health post. Curative, preventive and promotional medical activities are carried out at the gerontology and geriatrics center, which is the reference medical structure of the area. This center, which is managed by the municipality of Dakar, receives logistical support from the Ministry of Health and Social Action (medical ambulance, medical equipment...). Under the Sesame Plan, all eligible and available benefits (consultations, essential drugs, para-clinical assessment, hospitalization) are in principle free of charge for the elderly.

\subsection{Type and Period}

This was a descriptive cross-sectional study that ran from May 09 to July 10, 2016 over a period of 62 days.

\subsection{Population Under Investigation}

The study included all the patients aged 60 and plus that were admitted to outpatient clinics in the various departments of the center. The patients had freely agreed to integrate this study. They were interviewed in French and during daytime from $9 \mathrm{am}$ to $12 \mathrm{pm}$, Monday to Friday. The interviews took 30 to 45 minutes per person. For those who spoke only Wolof (national language), a nurse from the center acted as interpreter. She had been trained on technical terms.

\subsection{Data Collection}

It was done by a doctoral student of medicine using a preestablished questionnaire which includes the following details: marital status, antecedents and field, current drug prescription, anthropometric constants, study of falls (number of falls in the last 3, 6 and 12 months, fall-related factors and complications).

\subsection{Data Analysis}

The parameters studied were recorded on a survey sheet. The analysis of the data was carried out with SPSS software 17.0 (statistical Package For Social Sciences). It was organized in two parts:

1. a descriptive part with the calculation of absolute and relative frequencies, with averages and standard deviations.

2. an analytical part with contingency study and frequency comparison. We used the Xi 2 (Chi-square) test or the Fisher test according to their application conditions at the significance level set at $\mathrm{p}<0.05$.

\section{Results}

\subsection{Descriptive Study}

\subsubsection{Sociodemographic Data}

The study population was 210 patients. All survey cards were used. Our population was composed of women (61\%), with an average age of 69 years (60 and 93 years) and a standard deviation of 7.45 . The age group between $60 / 70$ years was the most represented $(58 \%)$ while those aged over 80 years represented only $12 \%$. Married patients accounted for $72 \%$, widowed widows $20 \%$, divorced $5 \%$ and single persons 3\%. Retirement benefit recipients made up 37\% of our population and the majority of the patients $(92 \%)$ did not perform any activity.

\subsubsection{Clinical Data}

Of the patients, 54\% had normal weight, $20 \%$ were overweight, $16 \%$ were obese and $10 \%$ were underweight. The most common pathologies were visual acuity (70\%), arterial hypertension (52\%), osteoarthritis (25\%), type 2 diabetes (21\%), and Auditory acuity (14\%)... The proportion of patients practicing physical activity was $22 \%$, it consisted mainly in walking $92 \%$ but also cycling $4 \%$, swimming $3 \%$ and football $1 \%$. The most prescribed drug classes were antihypertensives (33\%), antidiabetics drugs (10\%) and analgesics $(13 \%) \ldots$ Regarding the therapeutic management, $42 \%$ of patients were on monotherapy, $12 \%$ on dual therapy, $21 \%$ under triple therapy, $2 \%$ under quad therapy, but $23 \%$ of patients received no treatment.

\subsubsection{The Data Regarding the Falls}

The frequency of the falls in patients was $27 \%$ (figure 1).

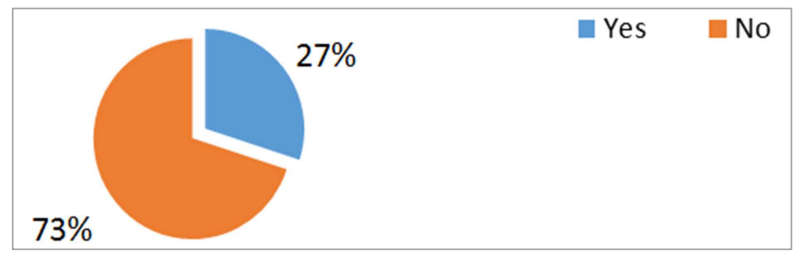

Figure 1. Prevalence of falls. 
Among the patients who had a fall, 18\% had had at least one fall during the last 3 months, $12 \%$ a fall during the last 6 months and $70 \%$ a fall during the last 12 months. According to the number of falls, $75 \%$ of the patients had a fall only once and $25 \%$ at least twice, of which $4 \%$ more than 5 times. The falls usually occurred at home (65\%) (Figure 2).

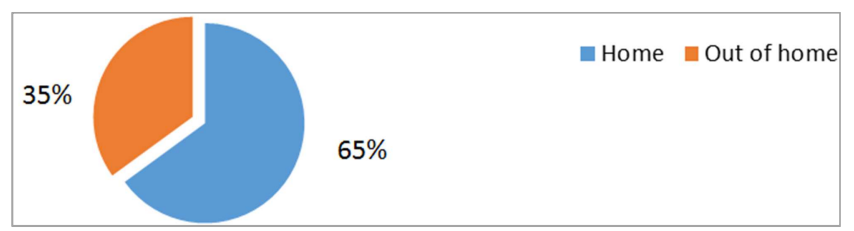

Figure 2. Place of falls.

The listed risk factors for falls were mainly soil barriers $(35 \%)$, loss of balance $(23 \%)$, wet surfaces $(16 \%)$, stairs $(9 \%)$, toilets $(3 \%)$, from bed $3 \%$, bad lighting $3 \% \ldots . .$. (Table 1 ).

Table 1. Breakdown by Falls Factors.

\begin{tabular}{lll}
\hline & Number & Percentage (\%) \\
\hline Extrinsic factors & & \\
a) Soil barriers & 22 & $35 \%$ \\
b) Wet surfaces & 10 & $16 \%$ \\
c) Stairs & 5 & $9 \%$ \\
d) Toilet & 2 & $3 \%$ \\
e) Bed & 2 & $3 \%$ \\
f) Bad lighting & 2 & $3 \%$ \\
g) Brawl & 1 & $2 \%$ \\
Intrinsic factors & & \\
a) Loss of balance & 16 & $23 \%$ \\
b) Visual blur & 1 & $2 \%$ \\
c) Stroke in the brain & 1 & $2 \%$ \\
d) Unspecified & 1 & $2 \%$ \\
\hline
\end{tabular}

All patients who had fallen had at least one complication. These were either psychological complications (mostly fear of falling again) or mechanical complications (mostly soft tissue bruises $62 \%$, injury $15 \%$, fractures $15 \%$ especially wrist fractures, sprains $8 \%$ ) (Table 2).

Table 2. Distribution of mechanical complications of falls.

\begin{tabular}{lll}
\hline & Number & Percentage (\%) \\
\hline Soft tissue bruises & 25 & $62 \%$ \\
Injury & 6 & $15 \%$ \\
Fractures & 6 & $15 \%$ \\
Wrist & 3 & $7 \%$ \\
Ankle & 2 & $5 \%$ \\
Forearm & 1 & $3 \%$ \\
sprains & 3 & $8 \%$ \\
Ankle & 2 & $5 \%$ \\
Knee & 1 & $3 \%$ \\
Total & 40 & 100 \\
\hline
\end{tabular}

\subsection{Analytical Study}

The analytical study did not find a statistical link between the falls and the risk factors studied, such as gender ( $\mathrm{p}=$ $0.28)$, age group $(\mathrm{p}=0.25)$, body mass $(\mathrm{p}=0.34)$, physical activity $(\mathrm{p}=0.89)$, therapeutic class administered $(\mathrm{p}=$ 0.756). However, the number of drugs administered at the time of the study is statistically incriminated for the occurrence of falls $(p=0.001)$.

\section{Discussion}

\subsection{Sociodemographic Data}

\subsubsection{Sex}

In our target population there was a female predominance (61\%). This overrepresentation of women is consistent with Senegal's current demographic data on the elderly [10]. Our study showed that women were more likely to fall $(82 \%)$. This female predominance was found in the Pradnya [11] and Hdiji [12] studies with prevalence's of $72 \%$ and $77 \%$, respectively. In contrast, Grundstorm [13] found a male predominance $(25 \%)$ in his study of patients over 85 years old. However, our study did not find a statistically significant relationship between sex and the number of falls $(p=0.28)$. However, the female sex is incriminated as a determining factor of falls in the elderly by some authors [11, 12, 14].

\subsubsection{Age}

The average age of our patients was 69 years. The age group between 60 and 70 years old accounted for $58 \%$ of patients and those over 80 years for $12 \%$. These rates are consistent with Senegal's national population data, which show a predominance of this age group in the elderly [10]. With regard to falls, 33\% of patients aged between 60 and 70 years and $7 \%$ of patients over 80 had a fall. Gelbard [15] also found that $47 \%$ of the persons victims of falls were between the ages of 80 and 90 in his study of falls among seniors. However, in our study, no significant statistical link was found between age groups and the number of falls $(\mathrm{p}=0.25)$. Yet the logic is that older people are more exposed to falls. The chances of falling and being seriously injured during a fall increase with age due to pathophysiological changes [16].

\subsubsection{Lifestyle and Physical Activity}

Sports activity was practiced by $22 \%$ of our population, and walking accounted for $91 \%$. This rate is comparable to that obtained by Diallo [17] who found that $80 \%$ of the patients had walked once or several times a week. In fact, walking is a simple and accessible physical activity for the elderly, and it can be done in the neighborhood or even at home.

More than half of our fallers (64\%) did not practice physical activity, but no statistical link was established between physical activity and the number of falls. Indeed, physical activity prevents the appearance of a number of deleterious phenomena related to aging and generates in elderly subjects a physical well-being accompanied on the psycho-intellectual level of a feeling of satisfaction [17].

However, among the senior, physical activity plays an important role in maintaining physical function, cognition, and in the treatment of various chronic diseases [18]. If, with age, health problems are often the reason given for not practicing physical activity, it is important to emphasize that these reasons are precisely those that justify the need to 
encourage and support the practice of this physical activity. Indeed, lack of physical activity has been identified as one of the top three health behaviors with smoking and eating habits that together account for about $50 \%$ of overall mortality [19]. Walking speed is a marker of the elderly person's state of health and a good indicator of his mobility.

\subsection{Anthropometric Data}

In our study, the prevalence of obesity was $15 \%$ and overweight at $20 \%$. Among seniors who had fallen, $25 \%$ were overweight or obese. However, in our study, there was no significant statistical link between the body mass index and the number of falls $(\mathrm{p}=0.34)$, contrary to the results of the Pin study [20]. Overweight not only causes instability in a static condition but also during the initiation of walking, resulting in less control of the center of gravity dynamics in the vertical plane, thus increasing the risk of falls, fractures and morbidity lower limbs [14].

\subsection{Clinical Data}

\subsubsection{Visual Disorders}

In our survey, $70 \%$ of the older people had experienced decrease in their visual acuity. Sight disorders in the elderly are very common. Sight in human beings evolves naturally towards presbyopia, due to the gradual loss of suppleness of the lens with age. These disorders of vision in the elderly related to the normal aging process of the eye are aggravated by certain systemic or metabolic diseases. If presbyopia increases with age, other disorders of vision in the elderly exist especially cataract. Among seniors who had a decrease in visual acuity $77 \%$ had fallen once. This prevalence of falls is much greater than the results of studies of Pin [20] and Minary [21].

This high figure in our study may be justified by the fact that some patients who had experienced decrease in sight acuity had financial difficulties to buy correction glasses, others did not find it useful to wear glasses or forgot to put them on by negligence. Knowing that wearing glasses reduces the rate of falls, not wearing them leads to a weakening of the faculties of avoidance due to bad judgment. Indeed, accurately estimating distances and perceiving spatial relationships is important for making the right decisions to travel [16]. We did not find a significant statistical link between the decrease in sight acuity and the number of falls $(\mathrm{p}=0.32)$.

\subsubsection{Cardiovascular Illnesses}

Hypertension (HTA) accounted for $52 \%$ of the pathologies encountered. This high prevalence of hypertension has been found in most studies [22, 23, 24, 25]. HTA is increasing and represents a real public health problem. Advanced age alone is a risk factor for this condition, especially when combined with obesity, stress, physical inactivity and a high-fat diet. In our study $75 \%$ of hypertensives had fallen. Gelbard [15] found a lower frequency (64\%) but a very significant link. This high rate recorded in our study should challenge practitioners on the management of high blood pressure.

\subsubsection{Balance and Walking Disorders}

The prevalence of osteoarthritis in our target population was $25 \%$ while the prevalence of balance and walking disorders was 28\%. Regarding disorders of balance and walking Yang [19] had found a frequency of $50 \%$ in the elderly. In the aging population, disorders of gait and balance represent a public health problem with an impairment of the quality of life.

If walking can be made difficult by an alteration of one or more coordinating functions, a precise semiological analysis would guide the syndromic and therefore etiological diagnosis. The severity of the disorders can be assessed using different clinical scales or specific tools that are rarely used [26].

Of the patients with osteoarthritis, $71 \%$ had a one-time fall and those with walking and balance disorders, $88 \%$ had fallen once. A frequency almost identical to that of Tsai et al [27] (82\%) and much higher than that of Pradnya D et al [11] (24\%) who found a significant link between these disorders and falls. Gait and balance disorders and osteoarthritis have been consistently identified in multiple studies, among the most important risk factors for falls. The pattern of walking in older people tends to be more rigid and less coordinated with poor postural control. Body orientation, reflexes, muscle strength and tone, as well as the length and height of walking decrease with aging and compromise the ability to avoid a fall [16].

\subsection{Therapeutic Management}

The most prescribed drugs were hypotensives $(33 \%)$, oral antidiabetics drugs (10\%), analgesics (9\%), antiinflammatory $(5 \%) \ldots$ Among patients who took antihypertensives, $60 \%$ had fallen, this frequency was higher than that found by Minary [22] (44\%), Montali [28] (32\%) and Tsai [27] (58\%). Of the patients taking oral antidiabetics drugs, 43\% had fallen. Montali [28] and Minary [22] found lower rates respectively $32 \%$ and $13 \%$ respectively. By cons Tsai [27] had found 50\%. These high frequencies in our study could be justified by the use of certain drugs incriminated in the fall risk such as digoxin, anti-arrhythmics and diuretics... especially that half of the patients was suffering from cardiovascular pathologies.

Physicians should be careful because there is an increased risk of falls the day after a prescription [16]. Buatois [14] accused poly-medication as a risk factor for falls. A consumption of more than four drugs was a criterion in his simple clinical scale to assess the risk of repeated falls among seniors. However, in our study, patients who took more than 4 medications accounted for only $2 \%$ of fallers. Minary [22] found a much higher frequency $(16 \%)$. This low rate in our study could be justified by the modest size of our study population.

\subsection{Study of Falls}

\subsubsection{Prevalence}

The frequency of the fall in our population was $27 \%$. This 
proportion is almost identical to that found by Pradnya D [11] (29\%). However it remains in deca of $76 \%$ of Hdiji [12], $45 \%$ of Grundstorm [13], $47 \%$ of Gelbard [15] and $38 \%$ of Pin [20].

It should be noted that the study of Hdiji [12] concerned falls of neurological origin. In the case of falls, frequencies higher than those found in Senegal were recorded in France (40\%), China (44\%) and India (53\%) [11, 16, 29]. This could be explained by the sociocultural context of our societies where the extended family continues to play a supporting role in helping and accompanying the elderly person in his daily activities (walking, cooking...). In fact, reckless activities are involved in more than a quarter of falls [14].

\subsubsection{Frequency and Number of Falls}

It was noted that $25 \%$ the patients had fallen at least 2 times in the last twelve months. Tsai [27] had found 35\% and Buatois [14] (39\%) in a study on the establishment of a simple clinical scale to assess the risk of repeated falls among the senior. However, some patients had fallen only once in the last three months $(18 \%)$. This is almost identical to the rate found by Grundstrom [13] (16\%).

\subsubsection{Falls Locations}

The majority of falls occurred at home $(64 \%)$ in our study as in other studies $[4,21]$. These figures could be justified by the fact that the senior spend most of their time at home.

\subsubsection{Risk Factors for Falls}

Extrinsic factors: Extrinsic factors were represented mainly by the obstacles on the ground (35\%) and the wet ones $(16 \%)$. These obstacles were composed of stones (outside and inside), stools or furniture that made patients stumble. As for wet soils, they are mainly due to the presence of puddles in the environment. These puddles may have been caused by inadvertent liquid substances (water, coffee, tea, juice...) or splashing due to the strong jets of the faucets. Pin [21] found the same extrinsic factors in his study, tripping and slipping (95\%).

Intrinsic factors: The equilibrium that is found in a state of stability in a standing position is the neutral result of the encounter of two forces: gravity (which attracts us to the ground) and muscular contractions (which keep us vertically). Thus, the loss of balance corresponds to the difficulty of standing in the standing position. It can be accompanied by dizziness, fits of giddiness... In our study the loss of balance was the intrinsic factor that caused the greatest number of falls $(22 \%)$. This rate is almost identical to that of Pin [20] (21\%).

\subsubsection{The Complications Related to Falls}

Contusion of superficial soft tissue was the major mechanical complication (62\%); however, lower frequencies were found only in the Pradnya D [11] (25\%) and Pin [20] studies (44\%). Superficial injuries accounted for $15 \%$ of fallers, a frequency identical to that of Grundstorm [13] (14\%) but below the results of Hdiji [12] (25\%) and Pin [21] (18\%). The fractures concerned only $15 \%$ of the fallers.
These fractures were located at the wrist, ankle and forearm levels. Hdiji [12] found an almost identical frequency (14\%) and Pin [20] a much higher figure (33\%). This low rate could be justified by the fact that a fall is translated into a moderate pain that the old person bears without consulting. He/She sometimes waits for several days before agreeing to consult and discover the existence of a bone fracture or fracture. With age, the perception of pain changes and may delay judgment on the severity of the consequences of a fall. It should be noted that the fracture of the upper end of the femur, the most common fracture according to the literature, had not been found. All our patients feared falling again. This fear of falling is a common psychological consequence that results in decreased physical activity, increased isolation, altered social identity, depression, anxiety and reduced quality of life]. The falling phobia in the elderly usually develops after an actual or initiated fall [6]. This event can lead to an avoidance of walking manifested by a reduction of the perimeter of walking, or even a refusal to walk sometimes with the appearance of psychopathological complications. Indeed, some authors report the presence of a depressive syndrome in $39 \%$ of the elderly people with phobic disorders [6]. Somatic complications related to prolonged bed rest (pressure sores, bronchial super infection...) can also occur.

\section{Conclusion}

Falls from the elderly are a public health problem because of their frequency and severity. They are the leading cause of accidental death in this population. Any fall should be treated as a potentially serious condition and with a risk of recurrence and complications. The mechanism of the fall is most often multifactorial. Simple aging itself is a risk factor for falls, to which are added other diseases that cause disability. The majority of the falls took place at home. Extrinsic factors can also promote falls such as ground obstacles and the effects of medications administered. The bruises accounted for most of the mechanical complications, but all the patients developed the phobia of falling again.

At the end of this work, it is important to develop a national program to fight falls in the elderly based mainly on primary prevention and involving the beneficiaries (wearing glasses if necessary, practicing sport, having a balanced diet, regularly drinking water, observing the prescribed treatments...), families (putting the elderly person in a suitable and adapted environment, eliminating the risk of slipping, putting armrests and support points, reassuring the patient after a fall) and health professionals (coordinating drug prescriptions, correcting sight disturbances, balance and walking disorders, and under nutrition).

\section{References}

[1] OMS (2016): http://www.who.int/mediacentre/factsheets/fs344/fr/consulté en aout 2016 
[2] Ermanel C, Thélot B, Jougla E, Pavillon G (2007). Accidental mortality of everyday life in metropolitan French, 2000-2004 Bull Epidemiol Hebd: 318-322. https://www.cairn.info/revuefrancaise-des-affaires-sociales-2008-1-page-71.htm

[3] Bloch F (2016). Fall of the elderly person. EMC- Treaty of Medicine Akros: $\quad 10$ (3). http://dx.doi.org/10.1016/S1634/6939(15) 63057-5

[4] Dargent-Molina P, Bréart G (1995). Epidemiology of falls and fall-related injuries in the aged Rev Epidemiol Santé Publique: 43:

$72-83$. https://www.ncbi.nlm.nih.gov/labs/articles/7892520/

[5] Bloch F (2012). Critical falls: why remaining on the ground after a fall can be dangerous, whatever the fall J Am Geriatr Soc; $\quad 60$ : 1375-1376 https://www.safetylit.org/citations/index.php? fuse action...26

[6] Navarro Ocampo G, Bréjard V, Bonnet A (2016). Fall in the elderly: from psychological impact to psychic work. Rev $\begin{array}{lll}\text { NPG17 } & \text { (97): }\end{array}$ http://dx.doi.org/10.1016/j.npg.2016.02.003

[7] Fried LP, Tangen CM, Walston J, Newman AB, Hirsch C, Gottdiener J et al (2001). Frailty in older adults: evidence for a phenotype J Gerontol A Biol Sci Med Sci; 56: M146-M156. https://books.google.sn/books?isbn=1441969993 -

[8] Scheffer AC, Schulman's MJ, van Dijk N, van der Hooft T, de Rooij SE (2008). Fear of falling: measurement strategy, prevalence, risk factors and consequences among older persons Age Ageing: 37: 19-24 https://www.ncbi.nlm.nih.gov/pubmed/18194967

[9] Mbaye EM, Ridde V, Kâ O (2013).Good intentions are not enough: analysis of a health policy for the elderly in Senegal. Public health: 25 (1): 107-12 https://www.cairn.info/revuesante-publique-2013-1-page-107.htm

[10] Ministère de l'Economie et des Finances du Sénégal (2002). National Agency of Statistics and Demography (ANSD). Third General Census of the Human Population of Senegal. Chap. 10: 119-131. www.ansd.sn/ressources/rapports/RGPH3_RAP_NAT.pdf

[11] Pradnya D, Ragupathy Sendhilkumar MSc, MPT (2016). Prevalence of risk factors for falls among elderly people living in long-term care homes. Journal of Clinical Gerontology Geriatrics: $\quad 7 \quad$ (3): $\quad 99-103$ http://dx.doi.org/10.1016/j.jcgg.2016.03.004consulté en mai 2016

[12] Hdiji O, Damak M, Bouzidi N, MhiriC (2016). Risk of Fails due to neurologic factors in an elderly Tunisian population. http://dx.doi.org/10.1016/j.npg.2016.03.001 consulté en juin 2016

[13] Grundstrom AC, Guse CE, Layde PM (2012). Risk factors for falls and fall-related injuries in adults 85 years of age and older. Archives of Gerontology and Geriatrics 54: 421-428

[14] Buatois S (2011). Establishment of a simple clinical scale to assess the risk of repeated falls in seniors Kinesither Rev: (111): 45-48. xemtailieu.com > Thểloạikhác > Chưaphânloại

[15] Gelbard R, Inaba K, Okoye OT, Morrell M, Saadi Z, Lam L et al (2014). Falls in the elderly: a modern look at an old problem The American Journal of Surgery: 208, 249-253. http://dx.doi.org/10.1016/j.amjsurg.2013.12.034

[16] Ambrose AF et al (2013). Risk factors for falls among older adults: A review of the literature Maturitas 75: 51-61. http://dx.doi.org/10.1016/j.maturitas.2013.02.009

[17] Waldron N, Dey I, Nagree Y et al (2011). A multi-faceted intervention to implement guideline care and improve quality of care for older people who present to the emergency department with falls BMC Geriatr, 11, p. 6 bmcgeriatr.biomedcentral.com/articles/10.1186/1471-2318-11-6

[18] Ciangura C, Faucher P, Oppert J-M (2014). Physical activity, nutrition and obesity. Clinical nutrition and metabolism 28: 279-286 www.em-consulte.com/article/942174/activitephysique-nutrition-et-obesite

[19] Yang X. J. et al (2011). Balance concerns in the elderly: Real or imaginary? / Journal of Clinical Gerontology \& Geriatrics 2: $109-115$ https://www.researchgate.net/.../230734240_Original_article

[20] Pin S, Vuillemin A, Léond C (2014). Prevalence of falls among people aged 55 to 85 and characteristics of fallers: results of the 2010 Health Barometer. 6th International Congress of Epidemiology / Journal of Epidemiology and Public Health: 62S-S213-S254. http://dx.doi.org/10.1016/j.respe.2014.06.273

[21] Minary A et al (2016). Drugs increasing the risk of falling: prospective study at Strasbourg University Hospital. Neurol psychiatr gériatr. http://dx.doi.org/10.1016/j.npg.2015.12.002

[22] Ka O, Mbaye E, Leye MMM, et al (2012). Sesam Plan in Senegal; limits of this model. Rev Public health: 24 (5): 439451.

[23] Fall F, Ka O, Diallo Y, Gaye A, et al (2015). Epidemiological profile of patients with erectile dysfunction at Ouakam health center, Dakar Senegal. Méd Afr Noire: 60 (11): 450-458

[24] Kane A (1995). Arterial Hypertension in Senegal: Epidemiological Aspects, Clinical Aspects and Problems of Therapeutic Management Dakar Medical, 40: 157-161. tropical-cardiology.com/.../25-1-hypertension-artérielle-enafrique-sub-saharienne-det..

[25] Thiam A (2010). Analysis of the consultation of the elderly received at the center of gerontology and geriatrics of Ouakam. Thèse Med, Dakar, Ucad 2010. number 83 http://bibnum.ucad.sn/viewer.php?c=thm\&d=THM-47223

[26] Chastan N, Welter M-L (2016). How do I examine a gait disorder? Neurological Practice - FMC. http://dx.doi.org/10.1016/j.praneu.2016.10.007consulté en aout 2016

[27] Tsai L-Y, Tsay S-L, Hsieh R-K, Yu S, Tsai J-M, Chien H-H, Liu S-J (2014). Fall Injuries and Related Factors of Elderly Patients at a Medical Center in Taiwan International Journal of Gerontology 203-208. http://dx.doi.org/10.1016/j.ijge.2013.10.007

[28] Montali F, Campaniello G, Benatti M, Rastelli, G, Pedrazzoni M, Cervellin G (2015). Impact of different drug classes on clinical severity of falls in an elderly population: Epidemiological survey in a trauma center Journal of Clinical Gerontology \& Geriatrics 6: 63-67. http://dx.doi.org/10.1016/j.jcgg.2015.03.002

[29] Hong-Ying P, Meng-Meng H, Jie Z, Pei-Pei P, Dan N (2015). Circumstances of falls and fall-related injuries among frail elderly under home care in China. International journal of $\begin{array}{lll}\text { nursing } & \text { sciences } & \text { 237-242. }\end{array}$ http://dx.doi.org/10.1016/j.ijnss.2015.07.002 